\title{
Análisis de la contaminación del Río Choluteca y sus efectos sobre la población a su paso por Tegucigalpa
}

Beatriz Ponce de Montoya*

\section{RESUMEN}

El creciente proceso de urbanización e industrialización que se está dando en el país promueve la contaminación de los principales sistemas fluviales del mismo. La contaminación deteriora la calidad del agua, por el cambio en sus características físicas, químicas y biológicas lo cual afecta negativamente la vida acuática, la salud humana, la producción, las actividades de la población y otras. La pérdida de la calidad del agua se debe a diversos factores entre ellos: erosión, arrastre de agroquímicos de los suelos, sedimentos, aumento poblacional, crecimiento urbano desordenado, eliminación inadecuada de heces fecales y desechos sólidos, mal estado o poca distribución de colectores de aguas negras y otros. Esto trae como consecuencia: Aumento de microorganismos patógenos que pueden provocar la muerte de personas y animales, proliferación de vectores acuáticos transmisores de enfermedades como el dengue, alteración de las propiedades del agua; $\mathrm{pH}$, turbidez, color, sabor, olor, etc., disminución de la diversidad biológica acuática, daños severos a los suelos y sus cultivos, eutrofización de las aguas lo que a su vez causa aumento de la demanda bioquímica de oxígeno, daños severos a la salud por contaminación por metales y otros.

La calidad del agua es un conjunto de características y propiedades de un cuerpo de agua que posibilita su uso con un fin determinado (Fundamentos Teóricos EducaciónAmbiental, 1999).

Se entiende por contaminación del agua al deterioro de su calidad por el cambio en sus características físicas, químicas o biológicas que afecta negativamente la vida acuática, la salud humana, las actividades de la sociedad, la infraestructura, la producción, etc.

Se considera que se genera contaminación en el agua por la adición de cualquier sustancia en cantidad suficiente para que cause efectos dañinos mensurables en la

*Beatriz Ponce de Montoya

Escuela de Biología, Universidad Nacional Autónoma de Honduras 
flora, la fauna (incluido al humano) o en los materiales de utilidad u ornamentales. Con frecuencia el sabor, el olor y el aspecto del agua indican que está contaminada, pero la presencia de contaminantes peligrosos sólo se puede detectar mediante pruebas químicas y biológicas específicas y precisas.

Existen valores máximos permisibles para parámetros ya sea físicos, químicos y biológicos, por ejemplo la presencia de nitrógeno amoniacal en el agua no debe exceder de $2.00 \mathrm{mg} / \mathrm{l}$, si sobrepasa este valor se considera contaminante (ver matriz de variables III.4).

En vista de las consecuencias que trae consigo la contaminación del agua de los ríos y haciendo una revisión de los estudios de contaminación realizados anteriormente y en proceso, estos enfocan más el análisis fisicoquímico con el objetivo de generar datos que sirvan para medir los efectos e influencia de las plantas depuradoras, entre otros. Por lo que el propósito de esta investigación será determinar el impacto de la contaminación sobre la población y qué medidas tomar ya sea para prevenir o mitigar tales efectos.

\section{Palabras Clave: Contaminación, Riesgo, Vulnerabilidad, Prevención, Mitigación}

\section{ABSTRACT}

The increasing process of urbanization and industrialization that is taking place in the country promotes contamination of the main fluvial system. Contamination deteriorates the quality of water due to the changes and its physical, chemical and biological characteristics which affect negatively the aquatic life, human health, production, population activities and others. The loss of the quality of water is due to different factors among them: erosion, agrochemical dragging, sediments, increase of population, urban disorganized growth, inappropriate elimination of feces and solid waste, bad condition or little distribution of sewage collectors and others. This brings as a consequence: growths of pathogen microorganism which can cause animal and human death, spreading of vectors, aquatic transmisors of diseases such as dengue fever alteration of properties of water, ph, blurriness, color, taste, odor, etc. Decreasing of the aquatic biological diversity, severe damages to the soil and crops eutrophication of the water which at the same time causes increase of the biochemistry demand of oxygen, harsh damages to health due to metal contamination and others.

The quality of water is a group of characteristics and properties of an open water 
body that facilitate its use with a specific purpose (Environment Education Theoric Fundaments, 1999).

It is understood for water contamination to the deterioration of the quality of water due to the changes in its characteristics, physical, chemical or biological that affect negatively the aquatic life, human health, and society activities, infrastructure, production, etc.

It is considered that contamination is generated in the water for the addition of any substances in enough amounts to cause some harmful measurable effects in the flora, fauna (including human beings) or in the usefulness or ornamental materials. Frequently the flavor, the odor and the water aspect show that it is contaminated, on the other hand dangerous pollutants only can be found trough precise and specific chemicals and biological tests.

There are maximum permissible values to parameters either to physical, chemical or biological, for instance the existence of ammonium-nitrogen in the water must not exceed $2.00 \mathrm{mg} / \mathrm{l}$, if it exceeds such rank is considered pollutant (see matrix of variables III.4)

Due to consequences that this comes with contamination of water in rivers revising contamination studies done formerly and in progress, these focus more on the physicochemical analysis with the purpose to generate data which will help measuring the effects and influence water treatment plant among others.

Which is why the purpose of this investigation will be to determine the impact of contamination on the population and which measurements to take either to prevent or mitigate such effects.

\section{Keyword: Contamination, risk, vulnerability, prevention, mitigation}




\section{INTRODUCCION}

La cuenca del Río Choluteca forma parte de la Vertiente del Pacífico, siendo la mayor de las cuencas que vierten sus aguas al Golfo de Fonseca. Estudios anteriores y recientes del Río Choluteca revelan que es un ecosistema sometido a una fuerte presión ambiental debido a que en sus márgenes se encuentran ubicados numerosos pueblos y ciudades como Tegucigalpa y Choluteca.

La calidad del agua es afectada por diferentes contaminantes que son introducidos a esta, a través de las descargas directas de aguas negras o por la escorrentía de aguas lluvias sobre la superficie de los suelos. Entre los principales contaminantes están: microorganismos patógenos, sólidos suspendidos (basura, partículas flotantes), agentes químicos (sustancias tóxicas, petróleo y derivados, nutrientes).

La presencia de microbios patógenos en el agua causa enfermedades como diarreas, hepatitis, poliomielitis y otras. Pueden provenir de aguas negras a través de la infiltración en aguas subterráneas someras o escorrentía de la lluvia en suelos contaminados con heces fecales u organismos en putrefacción.

La basura depositada en las riberas de los ríos es arrastrada por el agua acrecentando su contaminación. Existe una correlación entre el mal manejo del agua en reservorios, la eliminación inapropiada de residuos sólidos y la incidencia de enfermedades transmitidas por vectores como es el caso del Dengue que está afectando actualmente el territorio nacional.

En época de verano el problema de la contaminación en el agua se agrava por los olores nauseabundos que se producen al disminuir su caudal. Con el presente estudio se pretende hacer un análisis físico-químico y microbiológico del Río Choluteca a su paso por Tegucigalpa, identificando las principales fuentes, principales indicadores, los niveles actuales, y establecer los efectos de la contaminación como factores de riesgo en el ambiente. También se hará una investigación de campo utilizando encuestas que se aplicarán a la población que vive en las riberas del Río Choluteca a su paso por Tegucigalpa para identificar los efectos de la contaminación del mencionado río.

Para lograr los objetivos de estudio se identificarán los lugares de muestreo comprendidos entre la zona del puente Germania y el puente Juan Ramón Molina.

El análisis físico-químico y microbiológico consiste en el análisis de parámetros químicos, físicos y microbiológicos, para lo cual se tomaran muestras de agua de los puntos seleccionados para muestreo y los análisis se realizaran en el Centro de 
Estudios de Control y Contaminantes (CESCCO) siendo los parámetros químicos: Oxígeno disuelto, Demanda Biológica de Oxígeno, Demanda Química de Oxígeno, Nitrógeno, Fósforo.

Los parámetros físicos: Turbiedad, Conductividad, Sólidos disueltos, Color y los parámetros microbiológicos: Bacterias (coliformes totales y coliformes termoestables).

\section{METODOLOGÍA}

- Selección de zonas de muestreo del Río Choluteca en base a su accesibilidad, representatividad y costos, tomando en cuenta los puntos de monitoreo que utiliza el SANAA, siendo los puntos escogidos:

Punto N.1-Ala altura del Puente Germania.

Punto N.2-Ala altura del Puente El Prado.

Punto N.3-Ala altura del Puente Juan Ramón Molina.

- Toma de muestras de agua de las zonas escogidas, cada dos meses tanto en época lluviosa como en época seca ( a partir de agosto de 2005 a Febrero de 2006).

- Realizar evaluaciones físico-químicas y microbiológicas de las muestras de agua, tales como: Nitrógeno, Fósforo, Temperatura, Oxígeno disuelto y bacterias, para determinar el deterioro de la calidad de agua, complementando con los análisis de parámetros que el SANAA está realizando tales como: $\mathrm{pH}$, Demanda Bioquímica de Oxígeno (DBO), Demanda Química de Oxígeno (DQO), sólidos disueltos, color, conductividad, turbiedad, grasas y aceites.

- Identificación de las principales fuentes de contaminación del río consultando estudios anteriores y a través de visitas de campo basándose en la observación visual de las descargas puntuales y de desechos sólidos.

- Consultar bibliografías referentes a estudios que se han realizado sobre fuentes de contaminación en el país, así como bibliografía relacionadas con el tema.

- Conocer la cobertura y estado de los colectores de aguas negras sin tratamiento.

- Determinar el funcionamiento de plantas de tratamiento de aguas residuales en Tegucigalpa.

- Aplicar encuestas en casas y en fábricas aledañas a la orilla del Río Choluteca así como a personas que utilizan agua del río o trabajan en el mismo, para identificar los efectos de la contaminación de este río sobre la población, a su 
MATRIZ DE VARIABLES, INDICADORES E INDICES.

NORMAS DE CALIDAD PARA DESCARGA DE AGUAS RESIDUALES EN CUERPOS RECEPTORES

\begin{tabular}{|c|c|c|c|}
\hline VARIABLE & INDICADOR & $\begin{array}{l}\text { ÍNDICE Y UNIDAD } \\
\text { DE ANÁLISIS }\end{array}$ & INSTRUMENTO \\
\hline & & $\begin{array}{c}\text { (Concentración } \\
\text { máxima permisible) }\end{array}$ & \\
\hline \multirow{7}{*}{$\begin{array}{l}\text { Calidad del agua en } \\
\text { cuerpos receptores y } \\
\text { concentraciones } \\
\text { máximas permisibles } \\
\text { de descarga }\end{array}$} & Oxígeno disuelto & $\left(3 \mathrm{mg} / /^{\star}\right)$ & $\begin{array}{l}\text { Medición: } \\
\text { Oxínometro }\end{array}$ \\
\hline & $\mathrm{pH}$ & 6-9 NTU (4.5-9.5) & pHmetro \\
\hline & DBO & 50.00 mg/l. $\left(15 \mathrm{mg} / /^{\star}\right)$ & Método dilución \\
\hline & DQO & $200.00 \mathrm{mg} / \mathrm{l} .\left(50 \mathrm{mg} / \mathrm{I}^{*}\right)$ & $\begin{array}{l}\text { Método reflujo } \\
\text { cerrado. }\end{array}$ \\
\hline & Temperatura & $<25.00^{\circ} \mathrm{C}$ & $\begin{array}{l}\text { Visual con } \\
\text { termómetro }\end{array}$ \\
\hline & Fósforo total & $5.00 \mathrm{mg} / \mathrm{l}$ & Colorimetrico \\
\hline & Nitrógeno amoniacal & $20.00 \mathrm{mg} / \mathrm{l} .\left(2 \mathrm{mg} / \mathrm{I}^{\star}\right)$ & Nesslerización \\
\hline \multirow[t]{3}{*}{$\begin{array}{c}\text { Tipos de } \\
\text { Contaminantes }\end{array}$} & $\begin{array}{l}\text { Sólidos o partículas } \\
\text { suspendidas }\end{array}$ & $100.00 \mathrm{mg} / \mathrm{l}$ & Gravimétrico \\
\hline & $\begin{array}{c}\text { Materia flotante y } \\
\text { espuma }\end{array}$ & AUSENTE & $\begin{array}{l}\text { Visual con malla } \\
\text { flotante }\end{array}$ \\
\hline & $\begin{array}{c}\text { Organismos } \\
\text { patógenos: Bacterias } \\
\text { (coliforme fecal) }\end{array}$ & $5000 / 100 \mathrm{ml}$. & $\begin{array}{c}\text { Análisis } \\
\text { microbiológico: Tubos } \\
\text { múltiples o } \\
\text { Membrana filtrante }\end{array}$ \\
\hline $\begin{array}{l}\text { Fuentes productoras } \\
\text { de contaminación }\end{array}$ & $\begin{array}{c}\text { Fábricas de: } \\
\text { Alimentos } \\
\text { Bebidas }\end{array}$ & $\begin{array}{l}\text { Empacadoras de pollo } \\
\text { Bebidas carbonatadas }\end{array}$ & $\begin{array}{l}\text { Monitoreos } \\
\text { Monitoreos }\end{array}$ \\
\hline
\end{tabular}




\begin{tabular}{|c|c|c|c|}
\hline & $\begin{array}{l}\text { Hospitales } \\
\text { Otras }\end{array}$ & & Monitoreos \\
\hline \multirow{2}{*}{$\begin{array}{l}\text { Efectos sobre el } \\
\text { ambiente }\end{array}$} & Factores abióticos & Agua, suelo y aire & Monitoreos \\
\hline & Factores bióticos & $\begin{array}{l}\text { Seres vivos: flora y } \\
\text { fauna }\end{array}$ & Inventarios \\
\hline \multirow[t]{8}{*}{$\begin{array}{c}\text { Parámetros } \\
\text { indicadores de } \\
\text { contaminación }\end{array}$} & $\begin{array}{c}\text { Físicos: } \\
\text { pH }\end{array}$ & $\begin{array}{l}\text { Menor a } 4.5 \mathrm{mg} / \mathrm{l} . \text { y } \\
\text { mayor a } 9.5 \mathrm{mg} / \mathrm{l}^{*}\end{array}$ & Potenciómetro \\
\hline & Color & $>200 \mathrm{mg} / \mathrm{l}$ & Espectrofotométrico \\
\hline & $\begin{array}{c}\text { Químicos: } \\
\text { Grasas y aceites }\end{array}$ & $10 \mathrm{mg} / \mathrm{l}$ & Método de Extracción \\
\hline & $\begin{array}{l}\text { Demanda de } \\
\text { bioquímica de } \\
\text { oxígeno }\end{array}$ & $\begin{array}{l}\text { Mayor a } 50.00 \mathrm{mg} / \mathrm{l} \\
\left(\text { mayor a } 15 \mathrm{mg} / \mathrm{l}^{*}\right)\end{array}$ & Método de dilución \\
\hline & $\begin{array}{c}\text { Demanda química } \\
\text { de oxígeno }\end{array}$ & $\begin{array}{l}\text { Mayor a } 200 \mathrm{mg} / \mathrm{l} \text {. } \\
\left(\text { Mayor a } 50 \mathrm{mg} / \mathrm{l}^{\star} \text { ) }\right.\end{array}$ & Método de reflujo \\
\hline & Oxígeno disuelto & $0 \mathrm{mg} / \mathrm{l}$ & Oxinómetro \\
\hline & Conductividad & $\begin{array}{l}\text { Mayor a } 400 \mathrm{M} \\
\text { homnios } / \mathrm{cm}^{* *}\end{array}$ & Conductímetro \\
\hline & $\begin{array}{l}\text { Microbiológicos: } \\
\text { Bacterias fecales }\end{array}$ & Mayor a $5000 / 100 \mathrm{ml}^{*}$ & Análisis microbiológico \\
\hline
\end{tabular}

* Según la Propuesta Nacional de normas para agua de uso en preservación de flora y fauna (Calidad Básica del Agua).

** Según la norma técnica nacional para la calidad del agua potable.

\section{RESULTADOS DE LA INVESTIGACIÓN FISICOQUÍMICA Y MICROBIOLÓGICA}

\section{Tabulación de Datos}

Las muestras se tomaron en tres puntos del Río Choluteca a su paso por Comayaguela y Tegucigalpa y se analizaron en el laboratorio del Centro de Estudios y Control de Contaminantes (CESCCO) parámetros tales como DBO, DQO, grasas y aceites, nitrógeno amoniacal, fósforo total, coliformes totales, coliformes termotolerantes y el oxígeno disuelto (para el punto N. 3 correspondiente al Puente Juan Ramón Molina), los demás parámetros se analizaron en el laboratorio de control de calidad del SANAA. Para el análisis de datos se utilizó la metodología utilizada por esta institución. 


\section{Monitoreo del Río Choluteca (Período Lluvioso 2005 - Período Seco 2006)}

\begin{tabular}{|l|r|r|r|r|r|r|}
\hline \multicolumn{3}{|c|}{ ÉPOCA LLUVIOSA } & \multicolumn{3}{c|}{ ÉPOCA SECA } \\
\hline Temperatura del Agua & Ago-05 & Oct-05 & Promedio-05 & Dic-05 & Abr-06 & Promedio-06 \\
\hline Norma Técnica & & & & & & \\
\hline Puente Germania & & 20.8 & 20.8 & 21.8 & 27.4 & 24.6 \\
\hline Puente El Prado & & 21.2 & 21.2 & 22.3 & 26.7 & 24.5 \\
\hline Puente Juan R. Molina & & 22.1 & 22.1 & 24 & 27.5 & 25.7 \\
\hline
\end{tabular}

\begin{tabular}{|l|r|r|r|r|r|r|}
\hline \multicolumn{4}{|c|}{ ÉPOCA LLUVIOSA } & \multicolumn{3}{|c|}{ ÉPOCA SECA } \\
\hline \multicolumn{1}{|c|}{ Oxígeno Disuelto } & Ago-05 & Oct-05 & Promedio-05 & Dic-05 & Abr-06 & Promedio-06 \\
\hline Norma Técnica* & 3 & 3 & 3 & 3 & 3 & 3 \\
\hline Puente Germania & & $6.53^{\prime \prime}$ & 6.53 & $6.4^{\prime \prime}$ & $11.4^{\prime \prime}$ & 8.9 \\
\hline Puente El Prado & & $2.22^{\prime \prime}$ & 2.22 & 0 & 0 & 0 \\
\hline Puente Juan R. Molina & 4.4 & 2.2 & 3.3 & 0 & 0 & 0 \\
\hline
\end{tabular}

\begin{tabular}{|l|r|r|r|r|r|r|}
\hline \multicolumn{4}{|c|}{ ÉPOCA LLUVIOSA } & \multicolumn{3}{c|}{ ÉPOCA SECA } \\
\hline Coliformes Totales & Ago-05 & Oct-05 & Promedio-05 & Dic-05 & Abr-06 & Promedio-06 \\
\hline Norma Técnica* & 25,000 & 25,000 & 25,000 & 25,000 & 25,000 & 25,000 \\
\hline Puente Germania & 12,400 & 25,000 & 18,700 & 900 & 3,000 & 1,950 \\
\hline Puente El Prado & $6,300,300$ & $10,000,000$ & $8,150,000$ & $90,000,000$ & $60,000,000$ & $75,000,000$ \\
\hline Puente Juan R. Molina & $4,960,000$ & $10,000,000$ & $7,480,000$ & $95,000,000$ & $75,000,000$ & $85,000,000$ \\
\hline
\end{tabular}

\begin{tabular}{|l|r|r|r|r|r|r|}
\hline \multicolumn{4}{|c|}{ ÉPOCA LLUVIOSA } & \multicolumn{3}{c|}{ ÉPOCA SECA } \\
\hline Coliformes Termotolerantes & Ago-05 & Oct-05 & Promedio-05 & Dic-05 & Abr-06 & Promedio-06 \\
\hline Norma Técnica* & 5,000 & 5,000 & 5,000 & 5,000 & 5,000 & 5,000 \\
\hline Puente Germania & & 3,000 & 3,000 & 250 & 50 & 150 \\
\hline Puente El Prado & & $5,000,000$ & $5,000,000$ & $20,000,00025,000,000$ & $22,500,000$ \\
\hline Puente Juan R. Molina & $3,000,000$ & $3,000,000$ & $5,000,000$ & $12,500,000$ & $87,500,000$ \\
\hline
\end{tabular}

\begin{tabular}{|l|r|r|r|r|r|r|}
\hline \multicolumn{4}{|c|}{ ÉPOCA LLUVIOSA } & \multicolumn{3}{c|}{ ÉPOCA SECA } \\
\hline Nitrógeno Amoniacal & Ago-05 & Oct-05 & Promedio-05 & Dic-05 & Abr-06 & Promedio-06 \\
\hline Norma Técnica* & 2 & 2 & 2 & 2 & 2 & 2 \\
\hline Puente Germania & 0.35 & 0.64 & 0.495 & 0.37 & 1.12 & 0.74 \\
\hline Puente El Prado & 40.3 & 1.95 & 2.99 & 12.7 & 35.48 & 24.09 \\
\hline Puente Juan R. Molina & 2.09 & 1.95 & 2.425 & 11.2 & 29.78 & 20.49 \\
\hline
\end{tabular}




\begin{tabular}{|l|r|r|r|r|r|r|}
\hline \multicolumn{4}{|c|}{ ÉPOCA LLUVIOSA } & \multicolumn{3}{c|}{ ÉPOCA SECA } \\
\hline \multicolumn{1}{|c|}{ Fósforo Total } & Ago-05 & Oct-05 & Promedio-05 & Dic-05 & Abr-06 & Promedio-06 \\
\hline Norma Técnica** & 5 & 5 & 5 & 5 & 5 & 5 \\
\hline Puente Germania & 0.09 & 0.21 & 0.15 & 0.34 & 0.67 & 0.504 \\
\hline Puente El Prado & 0.6 & 1.5 & 1.05 & 42.7 & 6.758 & 5.72 \\
\hline Puente Juan R. Molina & 0.47 & 1.5 & 0.985 & 4 & 6.49 & 5.24 \\
\hline
\end{tabular}

\begin{tabular}{|l|r|r|r|r|r|r|}
\hline \multicolumn{4}{|c|}{ ÉPOCA LLUVIOSA } & \multicolumn{3}{c|}{ ÉPOCA SECA } \\
\hline \multicolumn{1}{|c|}{ Aceites y Grasas } & Ago-05 & Oct-05 & Promedio-05 & Dic-05 & Abr-06 & Promedio-06 \\
\hline Norma Técnica** & 10 & 10 & 10 & 10 & 10 & 10 \\
\hline Puente Germania & 5.1 & 1.3 & 3.2 & $<0.1$ & N.D & $<0.1$ \\
\hline Puente El Prado & 6.5 & 4.5 & 5.5 & 14 & N.D & 14 \\
\hline Puente Juan R. Molina & 5.1 & 3.3 & 4.2 & 9.4 & N.D & 9.4 \\
\hline
\end{tabular}

\begin{tabular}{|l|r|r|r|r|r|r|}
\hline \multicolumn{4}{|c|}{ ÉPOCA LLUVIOSA } & \multicolumn{3}{c|}{ ÉPOCA SECA } \\
\hline \multicolumn{1}{|c|}{ DB05 } & Ago-05 & Oct-05 & Promedio-05 & Dic-05 & Abr-06 & Promedio-06 \\
\hline Norma Técnica** & 50 & 50 & 50 & 50 & 50 & 50 \\
\hline Puente Germania & 1.8 & 5 & 3.4 & 5 & 3.80 & 4.4 \\
\hline Puente El Prado & 3.5 & 4.5 & 15.65 & 91 & 87.80 & 89.4 \\
\hline Puente Juan R. Molina & 7.6 & 3.3 & 16.3 & 76 & 83.80 & 79.9 \\
\hline
\end{tabular}

\begin{tabular}{|l|r|r|r|r|r|r|}
\hline \multicolumn{4}{|c|}{ ÉPOCA LLUVIOSA } & \multicolumn{3}{c|}{ ÉPOCA SECA } \\
\hline \multicolumn{1}{|c|}{ DQO } & Ago-05 & Oct-05 & Promedio-05 & Dic-05 & Abr-06 & Promedio-06 \\
\hline Norma Técnica** & 200 & 200 & 200 & 200 & 200 & 200 \\
\hline Puente Germania & 29 & 31 & 30 & 12 & 48 & 30 \\
\hline Puente El Prado & 51 & 70 & 60.5 & 214 & 280 & 247 \\
\hline Puente Juan R. Molina & 29 & 116 & 72.5 & 280 & 296 & 288 \\
\hline
\end{tabular}

\begin{tabular}{|l|r|r|r|r|r|r|}
\hline \multicolumn{4}{|c|}{ ÉPOCA LLUVIOSA } & \multicolumn{3}{c|}{ ÉPOCA SECA } \\
\hline \multicolumn{1}{|c|}{ Color } & Ago-05 & Oct-05 & Promedio-05 & Dic-05 & Abr-06 & Promedio-06 \\
\hline Norma Técnica** & $<200$ & $<200$ & $<200$ & $<200$ & $<200$ & $<200$ \\
\hline Puente Germania & 250 & 90 & 170 & 55 & 35 & 45 \\
\hline Puente El Prado & 225 & 75 & 150 & 350 & 1,250 & 800 \\
\hline Puente Juan R. Molina & 225 & 70 & 16.3 & 300 & 1,750 & 1,025 \\
\hline
\end{tabular}

\begin{tabular}{|l|r|r|r|r|r|r|}
\hline \multicolumn{4}{|c|}{ ÉPOCA LLUVIOSA } & \multicolumn{3}{c|}{ ÉPOCA SECA } \\
\hline \multicolumn{1}{|c|}{ PH } & Ago-05 & Oct-05 & Promedio-05 & Dic-05 & Abr-06 & Promedio-06 \\
\hline Norma Técnica** & 6.9 & 6.9 & 6.9 & 6.9 & 6.9 & 6.9 \\
\hline Puente Germania & 7.31 & 7.51 & 7.41 & 7.80 & 8.38 & 8.09 \\
\hline Puente El Prado & 7.09 & 7.27 & 7.18 & 6.89 & 7.94 & 7.41 \\
\hline Puente Juan R. Molina & 6.9 & 7.12 & 7.01 & 6.98 & 8.27 & 7.6 \\
\hline
\end{tabular}




\begin{tabular}{|l|r|r|r|r|r|r|}
\hline \multicolumn{4}{|c|}{ ÉPOCA LLUVIOSA } & \multicolumn{3}{c|}{ ÉPOCA SECA } \\
\hline \multicolumn{1}{|c|}{ Conductividad } & Ago-05 & Oct-05 & Promedio-05 & Dic-05 & Abr-06 & Promedio-06 \\
\hline Norma Técnica & & & & & & \\
\hline Puente Germania & 50 & 96 & 73 & 180 & 260 & 220 \\
\hline Puente El Prado & 180 & 360 & 270 & 510 & 58 & 284 \\
\hline Puente Juan R. Molina & 130 & 370 & 500 & 480 & 510 & 495 \\
\hline
\end{tabular}

\begin{tabular}{|l|r|r|r|r|r|r|}
\hline \multicolumn{4}{|c|}{ ÉPOCA LLUVIOSA } & \multicolumn{3}{c|}{ ÉPOCA SECA } \\
\hline \multicolumn{1}{|c|}{ Turbiedad } & Ago-05 & Oct-05 & Promedio-05 & Dic-05 & Abr-06 & Promedio-06 \\
\hline Norma Técnica & & & & & & \\
\hline Puente Germania & 117 & 28.1 & 72.55 & 16.50 & 1.77 & 9.13 \\
\hline Puente El Prado & 102 & 20.5 & 61.25 & 146 & 122 & 134 \\
\hline Puente Juan R. Molina & 95.3 & 16.7 & 56 & 117 & 155 & 136 \\
\hline
\end{tabular}

\begin{tabular}{|l|r|r|r|r|r|r|}
\hline \multicolumn{4}{|c|}{ ÉPOCA LLUVIOSA } & \multicolumn{3}{c|}{ ÉPOCA SECA } \\
\hline \multicolumn{1}{|c|}{ Sólidos Disueltos } & Ago-05 & Oct-05 & Promedio-05 & Dic-05 & Abr-06 & Promedio-06 \\
\hline Norma Técnica & & & & & & \\
\hline Puente Germania & & 48 & 48 & & 130 & 130 \\
\hline Puente El Prado & & 180 & 180 & & 290 & 290 \\
\hline Puente Juan R. Molina & & 185 & 185 & & 255 & 255 \\
\hline
\end{tabular}

\begin{tabular}{|l|r|r|r|r|r|r|}
\hline \multicolumn{4}{|c|}{ ÉPOCA LLUVIOSA } & \multicolumn{3}{c|}{ ÉPOCA SECA } \\
\hline Sólidos Suspendidos & Ago-05 & Oct-05 & Promedio-05 & Dic-05 & Abr-06 & Promedio-06 \\
\hline Norma Técnica** & 100 & & & & & \\
\hline Puente Germania & & 12 & 12 & 4 & 12 & 8 \\
\hline Puente El Prado & & 8 & 8 & 172 & 424 & 298 \\
\hline Puente Juan R. Molina & & 28 & 28 & 124 & 628 & 376 \\
\hline
\end{tabular}

* Propuesta nacional de Normas para agua de uso en Preservación de Flora y Fauna.

** Norma de Calidad para Descarga de Agua Residuales en Cuerpos Receptores.

“ Dato tomado en el campo con el oxigenómetro 


\section{Análisis e Interpretación de los Datos}

Según los resultados de los análisis de laboratorio, del monitoreo realizado en el periodo lluvioso de 2005, se observa lo siguiente:

a. Puente Germania: Presenta valores promedio mínimos correspondiente a coliformes totales con 18,700 UFC/100 ml , coliformes termotolerantes $(3,000$ UFC $/ 100 \mathrm{ml})$, nitrógeno amoniacal $(0.495 \mathrm{mg} / \mathrm{l})$, fósforo total $(0.15 \mathrm{mg} / \mathrm{l})$, DBO $5(3.4 \mathrm{mg} / \mathrm{l}), \mathrm{DQO}(30 \mathrm{mg} / \mathrm{l})$ y aceites y grasas $(3.2 \mathrm{mg} /)$ y cuenta con un valor promedio máximo para oxigeno disuelto con $6.53 \mathrm{mg} / \mathrm{l}$.

b. Puente EI Prado: Presenta valores máximos y fuera de norma para coliformes totales $(8,150,000 \mathrm{UFC} / 100 \mathrm{ml})$, coliformes termotolerantes $(5$, $000,000 \mathrm{UFC} / 100 \mathrm{ml})$ y nitrógeno amoniacal $(2.99 \mathrm{mg} /)$. Los valores promedio mínimos son para oxígeno disuelto con $2.22 \mathrm{mg} / \mathrm{l}$ y para sólidos suspendidos con $8 \mathrm{mg} / \mathrm{l}$.

c. Puente Juan Ramón Molina: Se observa valores fuera de norma para coliformes totales con 7, 480,000 UFC/100 ml, coliformes termotolerantes con $3,000,000 \mathrm{UFC} / 100 \mathrm{ml}$, nitrógeno amoniacal con $2.42 \mathrm{mg} / \mathrm{l}$ y oxígeno disuelto con $3.3 \mathrm{mg} / \mathrm{l}$. Se observa un valor promedio mínimo para color con $148 \mathrm{UC}$.

\section{Cuadro N.2 Valores promedio del Monitoreo del Río Choluteca Periodo Lluvioso 2005}

\begin{tabular}{|c|c|c|c|c|}
\hline Parámetros de Muestreo & Norma & $\begin{array}{l}\text { 1. Puente } \\
\text { Germania }\end{array}$ & $\begin{array}{l}\text { 2. Puente El } \\
\text { Prado }\end{array}$ & $\begin{array}{l}\text { 3. Puente Juan } \\
\text { R. Molina } \\
\end{array}$ \\
\hline Temperatura del Agua $\left({ }^{\circ} \mathrm{C}\right)$ & & 20.8 & 21.2 & 22.1 \\
\hline Oxígeno Disuelto & $3^{*}$ & 6.53 & 2.22 & 3.3 \\
\hline Coliformes Totales (UFC/100 mlL) & $25,000^{*}$ & 18,700 & $8,150,000$ & $7,480,000$ \\
\hline Coliformes Termotolerantes (UFC/100 ml) & $5,000^{*}$ & 3,000 & $5,000,000$ & $3,000,000$ \\
\hline Nitrógeno Amoniacal (mg/l) & $2^{*}$ & 0.495 & 2.99 & 2.425 \\
\hline Fósforo Total (mg/l) & $5^{* \star}$ & 0.15 & 1.05 & 0.98 \\
\hline $\mathrm{DBO}(\mathrm{mg} / \mathrm{l})$ & $50^{* *}$ & 3.4 & 15.6 & 16.3 \\
\hline $\mathrm{DQO}(\mathrm{mg} / \mathrm{l})$ & $200^{* *}$ & 30 & 60.5 & 72.5 \\
\hline Aceites y Grasas (mg/l) & $10^{* *}$ & 3.2 & 5.5 & 4.2 \\
\hline Color (UC) & $<200^{* *}$ & 170 & 150 & $\overline{148}$ \\
\hline Sólidos Suspendidos (mg/l) & $100^{* *}$ & 12 & 8 & 28 \\
\hline $\mathrm{pH}$ & $6-9^{* *}$ & 7.41 & 7.18 & 7.01 \\
\hline Sólidos Disueltos (mg/l) & & 48 & 180 & 185 \\
\hline Conductividad (Mhoms/cm) & & 73 & 270 & 500 \\
\hline Turbiedad (NTU) & & 72.55 & 61.25 & 56 \\
\hline
\end{tabular}

Valor fuera de norma Valor máximo y fuera de norma Valor mínimo
* Propuesta de normativa de uso del agua en preservación de flora y fauna.

** Norma técnica de descarga de agua residual a cuerpos receptores y alcantarillado sanitario. 
Según los resultados de los análisis de laboratorio, del monitoreo realizado en el periodo seco de 2006, se observa lo siguiente:

a. Puente Germania: Presenta valores promedio mínimos para coliformes totales (1,950 UFC/100 ml), coliformes termotolerantes (150 UFC/ $100 \mathrm{ml})$, nitrógeno amoniacal $(0.74 \mathrm{mg} / \mathrm{l})$, fósforo total $(0.50 \mathrm{mg} / \mathrm{l})$, aceites y grasas (< $0.1 \mathrm{mg} / \mathrm{l}), \mathrm{DBO} 5(4.4 \mathrm{mg} / \mathrm{l}), \mathrm{DQO}(30 \mathrm{mg} / \mathrm{l})$, color (45 UC) y un valor promedio máximo para oxigeno disuelto $(8.9 \mathrm{mg} / \mathrm{l})$.

b. Puente EI Prado: Presenta valores promedio fuera de norma para coliformes totales $(75,000,000 \mathrm{UFC} / 100 \mathrm{ml})$, coliformes termotolerantes $(22,500,000$ UFC/ml), DQO (247 mg/l), aceites y grasas (14 mg/l), color (800 UC) y sólidos suspendidos $(298 \mathrm{mg} / \mathrm{l})$. Los valores promedio máximos y fuera de norma corresponden a nitrógeno amoniacal $(24.09 \mathrm{mg} / \mathrm{l})$, fósforo total $(5.72 \mathrm{mg} / \mathrm{l})$, DBO5 (89.4 mg/l). Se observa que el oxigeno disuelto presenta valores de 0 $\mathrm{mg} / \mathrm{l}$.

c. Puente Juan Ramón Molina: Los parámetros que presentan valores promedios máximos y fuera de norma corresponden a coliformes totales (85, $000,000 \mathrm{UFC} / 100 \mathrm{ml}$ ), coliformes termotolerantes (87, 500,000 UFC/ml), DQO (288 mg/l), color (1,025 UC) y sólidos suspendidos (376 mg/l). Se observan valores fuera de norma para nitrógeno amoniacal $(20.49 \mathrm{mg} / \mathrm{l})$, fósforo total (5.24 mg/l), DBO5 (79.9 mg/l). En este punto también se observa que el oxígeno disuelto presenta valores de $0 \mathrm{mg} / \mathrm{l}$.

\section{Cuadro N.3 Valores promedios del Monitoreo del Río Choluteca Periodo Seco 2006}

\begin{tabular}{|c|c|c|c|c|}
\hline \begin{tabular}{|ll} 
Parámetros de Muestreo & Puntos de Muestreo \\
\end{tabular} & Norma & $\begin{array}{l}\text { 1. Puente } \\
\text { Germania }\end{array}$ & $\begin{array}{l}\text { 2. Puente EI } \\
\text { Prado }\end{array}$ & $\begin{array}{l}\text { 3. Puente Juan } \\
\text { R. Molina }\end{array}$ \\
\hline Temperatura del Agua $\left({ }^{\circ} \mathrm{C}\right)$ & & 24.6 & 24.5 & 25.7 \\
\hline Oxígeno Disuelto (mg/l) & $3^{*}$ & 8.9 & 0 & 0 \\
\hline Coliformes Totales (UFC/100 mlL) & $25,000^{*}$ & 1,950 & $75,000,000$ & $85,000,000$ \\
\hline Coliformes Termotolerantes (UFC/100 ml) & $5,000^{*}$ & 150 & $22,500,000$ & $87,500,000$ \\
\hline Nitrógeno Amoniacal (mg/l) & $2 *$ & 0.74 & 24.09 & 20.49 \\
\hline Fósforo Total (mg/l) & $5^{* *}$ & 0.5 & 5.72 & 5.24 \\
\hline $\mathrm{DBO}(\mathrm{mg} / \mathrm{l})$ & $50^{* *}$ & 4.4 & 89.4 & 79.9 \\
\hline $\mathrm{DQO}(\mathrm{mg} / \mathrm{l})$ & $200^{* *}$ & 30 & 247 & 288 \\
\hline Aceites y Grasas (mg/l) & $10^{* \star}$ & $<0.1$ & 14 & 9.4 \\
\hline Color (UC) & $<200^{* *}$ & 45 & 800 & 025 \\
\hline Sólidos Suspendidos (mg/l) & $100^{* *}$ & 8 & 298 & 376 \\
\hline $\mathrm{pH}$ & $6-9^{* *}$ & 8.09 & 7.41 & 7.6 \\
\hline Sólidos Disueltos (mg/l) & & 130 & 290 & 255 \\
\hline Conductividad (Mhoms/cm) & & 220 & 284 & 495 \\
\hline Turbiedad (NTU) & & 9.13 & 134 & 136 \\
\hline
\end{tabular}




\section{Análisis del Monitoreo del Río Choluteca}

Al hacer el análisis de los resultados de laboratorio de las muestras de agua tomadas en el río Choluteca durante el periodo lluvioso de 2005 y periodo seco de 2006 se observa que existe una fuerte contaminación orgánica proveniente de aguas fecales, principalmente en el punto de muestreo N. 2 (Puente El Prado) seguido del punto N.3 (Puente Juan Ramón Molina), en los cuales el numero de coliformes totales y termotolerantes o fecales sobrepasan el valor límite; esto indica que hay descarga puntual de aguas negras (domesticas y/o municipales) no tratadas por lo que es de extrema necesidad de que los colectores de aguas negras tengan el debido mantenimiento y mayor cobertura así como la existencia de plantas depuradoras de aguas negras.

Los valores que se obtuvieron para Nitrógeno amoniacal sobrepasaron la norma técnica $(2 \mathrm{mg} / \mathrm{l})$ en el punto N.2 y el punto N.3 como resultado de la descomposición de materia fecal.

En base a estos resultados se observa que la contaminación en el río Choluteca es predominantemente por descargas puntuales (aguas negras sin tratamiento). También se observa que los valores de DBO Y DQO se presentan fuera de norma en los puntos N.2 y N.3 durante el periodo seco de 2006.

Existen dos fábricas antes del punto N.2 y N.3 de las cuales una dijo arrojar sus desechos líquidos por la tubería de alcantarillado y la otra según fuentes fidedignas no aplican tratamiento a sus aguas residuales y son depositadas al río, contribuyendo así a que haya mayor concentración de materia orgánica e inorgánica.

Se observó durante los muestreos realizados en el río Choluteca la presencia de un botadero de desechos sólidos (basura) en el punto N.3 correspondiente al Puente Juan Ramón Molina, a orillas del río en mención. Esto representa un foco de contaminación principalmente en época lluviosa ya que por efecto de escorrentía puede llevar contaminantes al río.

\section{a. Identificación de las principales industrias de contaminación del Río Choluteca a su paso por Tegucigalpa y Comayaguela}

En 1996 el Centro de Estudios y Control de Contaminantes (CESCCO) identificó las principales industrias de contaminación del Río Choluteca a su paso por Tegucigalpa y Comayagüela siendo mayormente las industrias de producción de alimentos las cuales generan efluentes con contenido significativo de materia orgánica CESCCO detectó que de las treinta y dos (32) industrias visitadas en la Región Metropolitana, diez (10) de ellas carecen de sistemas de tratamiento de aguas residuales, una (1) dispone inadecuadamente sus efluentes y una (1) cuenta con tratamiento deficiente. 
También identificó otro problema ambiental como el manejo y disposición inadecuada de desechos sólidos, en la mayoría de los casos son enviados sin tratamiento al botadero municipal o son dispuestos directamente en las márgenes de ríos o quebradas y por arrastre llegan finalmente al Río Choluteca.

Así mismo hizo un estudio en nueve industrias de diferentes servicios tales como: alimenticias (de helados, de bebidas carbonatadas y procesadoras de pollo), químicas (revelado fotográfico, droguerías y fabrica de baterías para carro), de servicios (lavanderías), de la madera (fosforeras) y del concreto (fabrica de bloques). Los análisis revelaron que el $56 \%$ de las industrias evaluadas vierten sus efluentes al alcantarillado, el $44 \%$ vierten directamente al río Choluteca o uno de sus afluentes.

Todas las industrias se encuentran sobre los valores norma para los parámetros analizados de acuerdo a lo establecido en las Normas Técnicas para regular Descargas de Aguas Residuales a Cuerpos Receptores y Alcantarillados Sanitario (CTN-CALAGUA; 1997).

Los parámetros que se analizaron fueron $\mathrm{DBO}, \mathrm{DQO}$, nitrógeno amoniacal, nitritos, nitratos, fósforo total, cloruros, alcalinidad, sólidos suspendidos, sólidos sedimentables, metales pesados, hierro, níquel grasas y aceites y dureza. Las industrias que cuenta con un sistema de tratamiento primario son la industria alimenticia procesadora de pollos, la industria química de baterías para carro y la industria de fábrica de bloques, sin embargo no es suficiente para reducir los valores de algunos parámetros hasta cumplir con las normas técnicas.

La mayoría de las empresas industriales carecen de sistemas de tratamiento de aguas residuales y aunque la descarga no se realice directamente a un cuerpo receptor sino al alcantarillado sanitario, los efluentes tanto industriales como domésticos terminan incorporándose al río Choluteca.

Se visitó las oficinas de la Dirección de Evaluación y Control Ambiental (DECA) y de la Fiscalía del Ambiente para establecer las fuentes que contaminan el río Choluteca en la actualidad pero no existen denuncias notificando tales fuentes. Sin embargo se han identificado posibles fuentes tales como gasolineras, polleras, hospitales, mercados, imprentas, talleres, textilerias, galvanizadotas, entre otras. 
La Alcaldía Municipal a través de la unidad de gestión ambiental y el Comité de Emergencia Municipal (CODEM) realizan obras de prevención y mitigación de desastres tales como dragado y reforestación de ríos y quebradas.

\section{b. Análisis de la investigación Fisicoquímica y Microbiológica}

Los resultados de los análisis fisicoquímicos y microbiológicos demuestran que los puntos de muestreo que presentan mayor contaminación especialmente durante el periodo seco del 2006 corresponden a los puntos ubicados en el puente El Prado y en el puente Juan Ramón Molina, los valores de DBO y DQO sobrepasan el valor límite que es de 50 y de $200 \mathrm{mg} / \mathrm{l}$ respectivamente según la norma técnica de descarga de aguas residuales a cuerpos receptores y alcantarillado sanitario.

Esto indica que en el río se esta haciendo descarga de aguas residuales sin tratamiento provenientes de industrias.

Otros indicadores de contaminación que están por arriba de la norma son el color y los sólidos suspendidos, <200 UC y $100 \mathrm{mg} / \mathrm{l}$ respectivamente, el numero de los coliformes totales y termotolerantes esta alarmantemente fuera de norma (25,000 y 5,000 UFC/ $100 \mathrm{ml}$ respectivamente), lo cual es un indicativo de que hay una fuerte descarga puntual de aguas negras (domesticas y/o municipales) no tratadas, como resultado de la descomposición de la materia fecal se observa la presencia de Nitrógeno amoniacal con valores fuera de la norma, 2 $\mathrm{mg} / \mathrm{l}$ según la propuesta de normativa de uso del agua en preservación de la flora y fauna.

\section{c. Análisis del Trabajo de Encuestas}

Se aplicaron encuestas en los pobladores de las riberas del Río Choluteca como también a personas que trabajan en el río, a partir del puente Germania hasta la zona de La Isla, de 400 colonias en las riberas del río se seleccionaron las más cercanas, las encuestas en total fueron de 44.

La mayoría de las viviendas consultadas son de tipo marginal (84.85\%), se observa que el material de construcción predominante es el ladrillo (39.39\%), seguido de la madera (36.36\%), se observan casas construidas con laminas $(12.12 \%)$, otras son de madera y cartón $(9.09 \%)$ y un pequeño porcentaje es de bloque (3.03).

El $48 \%$ de la población presenta edades comprendidas entre los 15 a 50 años, la población que cuenta con edades entre 5 a 14 años es de $27 \%$,

el $11.5 \%$ de los habitantes presenta edades entre 1 a 4 años, el $7.5 \%$ son menores de un año, el $5.5 \%$ presenta edades mayores de 50 a 74 años.

La cobertura de acceso a agua potable en estos pobladores es de $69.7 \%$, el 30.3 $\%$ que no cuenta con este servicio directamente la obtiene de diferentes fuentes 
(vecinos y otros), las personas que tienen niños le dan tratamiento al agua que les dan de tomar, tales como hervirla o agregarle gotas de cloro como medida de precaución para evitar enfermedades gastrointestinales. Las enfermedades que más afectan a la población son de tipo respiratorio tales como gripes $(39.39 \%)$ y tos $(33.33 \%)$, los pobladores creen que esto es debido a los malos olores generados por el río Choluteca.

Respecto al servicio de recolección de basura el $66.66 \%$ de la población no cuenta con este servicio sin embargo la mayoría (63.63\%) elimina la basura quemándola. Los vectores que más afectan a la población son los zancudos $(55.88 \%)$ los cuales pueden transmitir enfermedades tales como el dengue, también un $47.05 \%$ de la población reportó tener problemas con ratas y ratones y un $38.23 \%$ dijo tener problemas con cucarachas, en suma el $20.58 \%$ de la población presentan en sus casas zancudos, ratas y ratones y cucarachas, todos transmisores de enfermedades.

El $73.68 \%$ de la población encuestada dijo no utilizar el agua del río ya que tiene acceso a agua potable y esta consciente que el río contiene aguas negras o sucias que pueden causar enfermedades, sin embargo existe un porcentaje (26.31\%) que se dedica a la extracción de arena, el 60\% de estas personas presentan afecciones en la piel, principalmente en los pies, que se caracteriza por picazón, ampollas y salpullido generalizado.

\section{CONCLUSIÓN}

La investigación realizada, contrario a lo que se espera con el grado de contaminación actual informa que son limitadas las enfermedades directas relacionadas con la contaminación del río, siendo los efectos principales que repercuten en la población principalmente los malos olores en época de verano, cuando las aguas residuales se encuentran en mayor concentración contaminante por la falta de aguas de lluvias.

Un segundo aspecto de interés sanitario es la presencia de vectores asociados tanto aéreos como rastreros, que en alguna oportunidad pueden afectar la salud de los habitantes de las riberas del río. El efecto paisajista ha sido mejorado por la canalización del río por parte de la alcaldía no obstante se observa indiferencia de la población al encontrarse promontorios de basura y materiales de construcción.

La mayormente afectada es la fauna hídrica la cual es prácticamente nula a lo largo del río Choluteca. 
Finalmente la amenaza de inundaciones es un elemento más relacionado con los pocos habitantes que aun viven en las orillas del río en situación de riesgo.

\section{RECOMENDACIÓN}

Como recomendación general se establece que el SANAA continúe construyendo las plantas depuradoras que faltan en la zona Guacerique, la zona El Estadio, la zona Río Chiquito entre otras; ampliar la cobertura de servicio de alcantarillado y el buen mantenimiento de los mismos en las zonas aledañas al río como también la necesidad de continuar con la canalización del Río Choluteca con sus niveles de flujo mínimo, medio y máximo con lo cual mejoraría también la estética paisajista en la columna vertebral del saneamiento de la ciudad capital.

En entrevista realizada al Ing. Rodolfo Ochoa de la DIAT del SANAA manifiesta que por razones económicas es prioritario realizar una canalización geométrica al centro del Río que transporte el agua residual en época de verano, con un segundo nivel de terraplén también geométrico en las márgenes del río requiriéndose para este propósito únicamente actividades de topografía y movimiento de tierra.

En una segunda etapa establecer la geometría necesaria para realizar la canalización en tres niveles para flujo mínimo promedio y flujo máximo. Finalmente proceder al enchape del río en sus tres niveles tal como se realiza en otras partes del mundo.

\section{AGRADECIMIENTOS}

Al Ing. Rodolfo Ochoa por brindarme su asesoría, a la Organización Panamericana de la Salud (OPS) por su apoyo financiero para los análisis de laboratorio y a la Dirección de Investigación Científica de la UNAH por su apoyo para la culminación de este estudio de tesis.

\section{BIBLIOGRAFÍA}

Agencia de Cooperación Internacional del Japón (JICA). 2002. Estudio sobre el Control de Inundaciones y Prevención de Deslizamientos de tierra en el Área Metropolitana de Tegucigalpa de la República de Honduras. Informe Final. Informe de Apoyo.

Andino Ana. 1986. Revisión Bibliográfica de los estudios realizados sobre la cuenca del Río Choluteca. Monografía. UNAH, Honduras. 
Balsiger. 1994. Contaminación del Río Choluteca por la ciudad de Tegucigalpa, Honduras. CESCOO-EPF.

Banco Interamericano de Desarrollo. 1999. Reducción de La Vulnerabilidad ante amenazas naturales. Tegucigalpa.

Caballero Zeitún, Elsa Lily. 2001. Construcción de Vulnerabilidad Humana. Resumen de Tesis Doctoral. Postgrado Latinoamericano en Trabajo Social, UNAH, Tegucigalpa.

Centro de Estudios y Control de Contaminantes (CESCCO).1996. Estudios de residuos industriales en Tegucigalpa, Honduras.

Centro de Estudios y Control de Contaminantes (CESCCO). 1997. Estudio de nueve efluentes en Tegucigalpa, Honduras.

Centro Panamericano de Ingeniería Sanitaria y Ciencias del Ambiente/ Organización Panamericana de la Salud. 2000. Prevención de la Contaminación de la pequeña y mediana Industria. Lima.

Clare Tatiana. 1982. Estudio de la contaminación del Río Choluteca a su paso por las ciudades de Comayagüela y Tegucigalpa, UNAH, Honduras.

Cruz G. 1987. Análisis de Macroinvertebrados Bénticos en el Río Choluteca y en dos de sus principales afluentes en Tegucigalpa.

Cruz G. y otros. 1987. Análisis de los Parámetros Fisicoquímicos asociados a macroinvertebrados bentónicos en el Río Choluteca y sus cabeceras.

CTN-CALAGUA-CAPRE. 1997. Normas Técnicas de Las Descargas de Aguas

Residuales a Cuerpos Receptores y Alcantarillado Sanitario. Tegucigalpa. Honduras.

División de Investigación y Asistencia Técnica (DIAT) del Servicio Autónomo Nacional deAcueductos y Alcantarillados.

Estudio de Rehabilitación Ambiental en Cuatro Subcuencas Hidrográficas del Sistema de Abastecimiento de Agua Potable para Tegucigalpa. 2005. Consultores ESAy Lotti y Asociados.

Manual de Disposición de Aguas Residuales. 1991. Cooperación Técnica República Federal de Alemania.

Manual para el Control de las Enfermedades Transmisibles. 1997. Organización Panamericana de la Salud.

Myton, Ponce et al. 1992. La Cuenca del Río Choluteca. Laboratorio de LimnologíaOEA, Departamento de Biología, UNAH.

Montoya Irasema. 1997. Fuentes de Contaminación de Cuerpos Receptoras de agua en Honduras. SANAA/OPS.

Ochoa Rodolfo et al 2001. Análisis del monitoreo del Río Choluteca. DIAT/SANAA. Ochoa Rodolfo et al 2002. Análisis del monitoreo del Río Choluteca, Periodo Seco y Periodo Húmedo. DIAT/SANAA.

Ochoa Rodolfo et al 2003. Análisis del Río Choluteca, Periodo Seco y Periodo 
Húmedo. DIAT/SANAA.

Ochoa Rodolfo et al 2004. Análisis del Río Choluteca, Período Seco y Período Húmedo. DIAT/SANAA.

Ochoa Rodolfo. 2004. Ficha Técnica N. 5, DIAT/SANAA.

Ochoa Rodolfo. 2005. Ficha Técnica N. 7, DIAT/SANAA.

Ortiz Pedro. 2006. Soluciones Técnicas para el tratamiento de Aguas Residuales en

Tegucigalpa. III Simposium de Ingeniería Ambiental "La Gestión del Riesgo en

Tegucigalpa".

Organización Panamericana de la Salud. 2004. Manual de Evaluación de daños y necesidades en salud para situaciones de desastre.

Paniagua Sergio. 2002. Desastres y Emergencias. Costa Rica.

Plataforma del Agua. 2004. Agua y Desarrollo. Tegucigalpa, Honduras.

Propuesta Nacional de Normas para Agua de Uso en Preservación de la Flora y

Fauna (Calidad Básica del Agua). 2001. CAPRE/Secretaria de Salud, Tegucigalpa, Honduras.

Revista del Colegio de Ingenieros Civiles de Honduras, Edición N. 9, Julio 2005. Rigola Miguel. 1989. Tratamiento de Aguas Residuales.

Secretaría de Salud/CAPRE. Borrador, 2001. Norma Técnica Nacional para Agua, Tegucigalpa, Honduras.

Tábora Fabiola. 2003. Diagnóstico rápido de la Cuenca del Río Choluteca. USAID. Unidad de Gestión Ambiental de la Alcaldía Municipal del Distrito Central, 2006.

www.cepis.ops-oms.org/enwww/eva2000/honduras/informe/inf-05.htm www.iadb.org/regions/re2/consultative_group/_vti_bin/shtml.

www.k12science.org/curriculum/dipproj/es/

www.lenntech.com/espanol/formulario-de-consulta.htm

http://njnie.dl.stevenstech.edu/curriculum/dipproj2/es/fielbook/oxigeno.shtml http://outreach.ecology.uga.edu/htm

www.cep.unep.org/pubs/techreports/tr40es/index.html

http://agua.ecoportal.net/nosotros

www.arqhys.com/construccion/septicas_fosas.html

www.purewaterplanet.com/sp/faq.htm

www.unicef.org/voy/spanish/explore/wes/explore_1917.html 